\title{
Evaluation of Muscle Mass and Stiffness with Limb Ultrasound in COVID-19 Survivors
}

OPEN ACCESS

Edited by:

Qing Feng Cheng,

First Affiliated Hospital of Chongqing

Medical University, China

Reviewed by:

Niharika Arora Duggal,

University of Birmingham,

United Kingdom

Karolina Maria Piotrowicz,

Jagiellonian University Medical

College, Poland

Tomasz Kostka,

Medical University of Lodz, Poland

${ }^{*}$ Correspondence:

Sarah Damanti

damanti.sarah@hsr.it

Specialty section:

This article was submitted to

Endocrinology of Aging,

a section of the journal

Frontiers in Endocrinology

Received: 24 October 2021

Accepted: 25 January 2022

Published: 17 February 2022

Citation:

Damanti S, Cilla M, Tuscano $B$,

De Lorenzo R, Manganaro G, Merolla A, Pacioni G, Pomaranzi C,

Tiraferri V, Martinenghi S, Vitali G,

Bosi E, Conte C, Giustina A, Tresoldi M

and Rovere Querini P (2022)

Evaluation of Muscle Mass and

Stiffness with Limb Ultrasound

in COVID-19 Survivors.

Front. Endocrinol. 13:801133.

doi: 10.3389/fendo.2022.801133

\begin{abstract}
Sarah Damanti ${ }^{1 *}$, Marta Cilla ${ }^{1}$, Bruno Tuscano ${ }^{2}$, Rebecca De Lorenzo ${ }^{3}$, Giuseppina Manganaro ${ }^{3}$, Aurora Merolla ${ }^{3}$, Giacomo Pacioni ${ }^{3}$, Chiara Pomaranzi ${ }^{3}$, Valeria Tiraferri ${ }^{3}$, Sabina Martinenghi ${ }^{4}$, Giordano Vitali ${ }^{4}$, Emanuele Bosi ${ }^{3,4}$, Caterina Conte ${ }^{5,6}$, Andrea Giustina ${ }^{7}$, Moreno Tresoldi ${ }^{1}$ and Patrizia Rovere Querini ${ }^{2,8}$

1 Unit of General Medicine and Advanced Care, Instituto di Ricovero e Cura a Carattere Scientifico (IRCSS) San Raffaele Institute, Milan, Italy, ${ }^{2}$ Unit of Radiology, IRCCS San Raffaele Institute, Milan, Italy, ${ }^{3}$ Vita-Salute San Raffaele University, Milan, Italy, ${ }^{4}$ San Raffaele Diabetes Research Institute, Instituto di Ricovero e Cura a Carattere Scientifico (IRCSS) Ospedale San Raffaele, Milan, Italy, ${ }^{5}$ Department of Human Sciences and Promotion of the Quality of Life, San Raffaele Roma Open University, Rome, Italy, ${ }^{6}$ Department of Endocrinology, Nutrition and Metabolic Diseases, Instituto di Ricovero e Cura a Carattere Scientifico (IRCSS) MultiMedica, Milan, Italy, ${ }^{7}$ Institute of Endocrine and Metabolic Sciences, San Raffaele VitaSalute University, Instituto di Ricovero e Cura a Carattere Scientifico (IRCSS) San Raffaele Hospital, Ospedale San Raffaele, Milan, Italy, ${ }^{8}$ Department of Immunology, Transplantation and Infectious Diseases, Instituto di Ricovero e Cura a Carattere Scientifico (IRCSS) Ospedale San Raffaele, Milan, Italy
\end{abstract}

Background: acute illnesses, like COVID-19, can act as a catabolic stimulus on muscles. So far, no study has evaluated muscle mass and quality through limb ultrasound in postCOVID-19 patients.

Methods: cross sectional observational study, including patients seen one month after hospital discharge for SARS-CoV-2 pneumonia. The patients underwent a multidimensional evaluation. Moreover, we performed dominant medial gastrocnemius ultrasound (US) to characterize their muscle mass and quality.

Results: two hundred fifty-nine individuals (median age 67, 59.8\% males) were included in the study. COVID-19 survivors with reduced muscle strength had a lower muscle US thickness (1.6 versus $1.73 \mathrm{~cm}, p=0.02$ ) and a higher muscle stiffness (87 versus 76.3, $p=$ 0.004) compared to patients with normal muscle strength. Also, patients with reduced Short Physical Performance Battery (SPPB) scores had a lower muscle US thickness (1.3 versus $1.71 \mathrm{~cm}, p=0.01$ ) and a higher muscle stiffness (104.9 versus 81.07, $p=0.04$ ) compared to individuals with normal SPPB scores. The finding of increased muscle stiffness was also confirmed in patients with a pathological value $(\geq 4)$ at the sarcopenia screening tool SARC-F (103.0 versus 79.55, $p<0.001$ ). Muscle stiffness emerged as a significant predictor of probable sarcopenia (adjusted OR 1.02, 95\% C.I. $1.002-1.04, p=0.03$ ). The optimal ultrasound cut-offs for probable sarcopenia were $1.51 \mathrm{~cm}$ for muscle thickness $(p=0.017)$ and 73.95 for muscle stiffness ( $p=0.004$ ).

Discussion: we described muscle ultrasound characteristics in post COVID-19 patients. Muscle ultrasound could be an innovative tool to assess muscle mass and quality in this population. Our preliminary findings need to be confirmed by future studies comparing muscle ultrasound with already validated techniques for measuring muscle mass and quality.

Keywords: muscle, ultrasound, sarcopenia, COVID - 19, muscle mass, muscle quality 


\section{BACKGROUND}

Sarcopenia is a progressive and generalized skeletal muscle disorder, characterized by an accelerated loss of muscle mass and function, which is associated with an increased likelihood of developing adverse outcomes (1-6). Decline in muscle mass is not homogenous across different body anatomic regions, as sarcopenia occurs earlier in the lower limbs (7). Moreover, muscle quality, which is precociously impaired in sarcopenia (2), has an important impact on muscle function (8) and clinical outcomes (9), independently from muscle mass reduction. Muscle quality is determined by micro- and macroscopic changes in muscle architecture and composition $(8,10)$. In research settings, magnetic resonance imaging (MRI) and computed tomography (CT) have been used to study muscle quality, by assessing fat infiltration into muscle, and evaluating muscle attenuation $(11,12)$. On the other hand, muscle quality has also been defined in functional terms, as the muscle strength delivered per unit of muscle mass $(13,14)$ or volume (15). Because of its effects on muscle performance and clinical outcomes, muscle quality should always be considered in the assessment of sarcopenic subjects. Unfortunately, there has not been universal consensus yet on which method should be used for the evaluation of muscle quality in the routine clinical practice (1).

Acute illnesses, like COVID-19, can act as a catabolic stimulus on muscles $(16,17)$. Indeed, weight loss has been reported to be pronounced in COVID-19 patients $(17,18)$ who are at high risk of developing acute sarcopenia $(19,20)$. However, the degree of muscle mass and functional loss depends on multiple factors: preexisting conditions (i.e. age, frailty, comorbidities), the degree of inflammatory response to the SARS-CoV-2 infection, anorexia, inadequate protein supply, and physical inactivity during the active phase of the COVID19 disease (21). Old, comorbid and frail patients are at higher risk of developing acute sarcopenia, even in the presence of a mild COVID-19 disease. Anyway, acute sarcopenia can occur in previously robust individuals too $(16,22,23)$.

Acute sarcopenia augments patients' vulnerability to stressors $(24,25)$, increasing their risk of developing adverse outcomes. Moreover, acute sarcopenia can evolve into chronic sarcopenia (26), a condition closely related to frailty (24).

Skeletal muscle ultrasound is an accurate imaging technique (27) for evaluating muscle architecture, and for quantifying muscle mass, as demonstrated by its comparison with direct anatomical assessment on cadavers $(28,29)$ and MRI studies (30). However, its role in diagnosing sarcopenia is just speculative. None of the current definitions of sarcopenia includes muscle echography in its diagnostic algorithms $(1,31$, 32), and the analysis of current medical literature highlights the absence of a standardized method for performing muscle ultrasonography, to detect sarcopenia in clinical practice (33). In addition, normative values for defining lower limb ultrasound quantity and quality are lacking.

Finally, no study has evaluated muscle mass in post COVID19 patients, through limb ultrasound, so far. The main objective of our study was evaluating muscle mass and quality through lower limb ultrasound in a cohort of COVID-19 survivors. As secondary objectives we performed i) a correlation of the muscle ultrasound parameters with validated measures of muscle function, nutritional status and inflammatory indexes during hospital stay, ii) an assessment of the association between muscle ultrasound parameters and probable sarcopenia and iii) a definition of ultrasound parameters cut-off values associated with probable sarcopenia.

\section{MATERIAL AND METHODS}

This was a cross sectional observational study. We evaluated patients attending a dedicated post-COVID-19 outpatient clinic, who were previously hospitalized for SARS-CoV-2 pneumonia in the Internal Medicine Departments of the San Raffaele University Hospital, Milan, Italy (34). The data presented in this study were collected in the visits that took place one month after hospital discharges, from the $15^{\text {th }}$ April 2021 till the $15^{\text {th }}$ July 2021 . We are still collecting data on three- and six-months follow-ups. The present study was part of the COVID-BioB study (NCT04318366), which aimed at characterizing hospitalized COVID-19 patients, through the prospective collection of several demographic, anthropometric, clinical and laboratory variables, as previously described (35). The COVID-BioB study protocol was approved by the San Raffaele University Hospital Ethics Committee (protocol no. 34/int/2020). A convenience sample size was used due to the setting of the COVID19 pandemic.

During the follow-up visits, the patients underwent a multidimensional evaluation, consisting in: medical history, including self-reported weight loss during hospitalization, physical examination, anthropometric measurements to calculate the body mass index (BMI), before hospital stay, and one month after hospital discharge, screening for sarcopenia through the Strength, Assistance with walking, Rising from a chair, Climbing stairs, and Falls (SARC-F) questionnaire (36), assessment of muscle strength through the hand grip strength test (37), evaluation of muscle performance with the Short Physical Performance Battery (SPPB) test (38) and screening for malnutrition with the Mini Nutritional Assessment Short Form (MNA-SF) questionnaire (39). Patients suffering from dementia were generally helped by their care-givers in the compilation of the questionnaires.

Finally, all patients underwent muscle ultrasound of the dominant medial gastrocnemius, to assess muscle mass and quality. We chose to evaluate gastrocnemius muscle, because it has a pennate architecture, thus allowing the assessment of pennation angle. Pennation angle is the angle formed at the fiber insertions into deep aponeurosis in pennate muscles and it is strongly correlated to muscle mass. Pennation angle was automatically calculated by the ultrasound software after the sonographer had manually identified the angle formed between muscle fiber insertions and deep aponeurosis.

By limb ultrasound, muscle quality can be assessed either with the determination of muscle echogenicity $(40,41)$, or with muscle stiffness (42). However, there is no consensus on which 
of these parameters should be preferred, in the assessment of muscle quality by limb ultrasound. Therefore, since our radiologists had more experience in the evaluation of muscle stiffness, we chose to investigate this aspect of muscle quality.

During muscle ultrasound, the patients laid prone on the examination couch, with the foot positioned perpendicularly to the tibia outside the couch. The ultrasound examinations were performed by two trained sonographers (SD and MC). To improve acoustic coupling, abundant water-soluble transmission gel was used on the linear array probe (7-10 $\mathrm{MHz}$, General Electric model), using B-mode. The probe was set perpendicularly to the dermal surface, to get images, including both superficial and deep aponeurosis, and with an orientation coinciding with that of the muscle fascicles between the aponeuroses. Images were obtained along the mid-sagittal line of the medial gastrocnemius at the mid-distance between its proximal and distal tendon insertions $(43,44)$. Depth was initially set at $30 \mathrm{~mm}$, then it was modified during the examination (range: $30-60 \mathrm{~mm}$ ) to visualize the entire muscle thickness. Resting Euclidean distance between the internal borders of the superficial and deep aponeuroses (i.e. muscle thickness) was assessed in three points of the muscle, equally spaced along the image, and a mean value was calculated. In addition, the angle between the fascicle and the deep aponeurosis (i.e. pennation angle) was calculated.

Images were stored as DICOM files, and transferred to a computer for processing. Muscle stiffness was measured by means of an AGFA Enterprise Imaging program. The size of regions of interest (ROI), to estimate the stiffness index (SI), was set between 0.2 and $0.3 \mathrm{~cm}^{2}$. ROI were measured in three points of the medial gastrocnemius, equally spaced along the images. Then a mean value of the three SI obtained, was calculated.

\section{Statistical Analyses}

The baseline characteristics of the study population, the main aspects of the COVID-19 hospitalization, muscle and nutritional parameters one month after hospital discharge were described through descriptive statistics. Continuous variables were presented as mean and standard deviation (SD), when normally distributed, or with median and interquartile range (IQR), when data had a skewed distribution. Dichotomous variables were presented as number $(\mathrm{N})$ and percentage (\%). In addition, we performed a comparison of the distribution of categorical and continuous variables among patients with reduced ( $\mathrm{SPPB} \leq 8)$ versus normal $(\mathrm{SPPB}>8)$ muscle performance, reduced (Hand Grip Strength $<27 \mathrm{~kg}$ in men or $<16 \mathrm{~kg}$ in women) versus normal (Hand Grip Strength $\geq 27$ $\mathrm{kg}$ in men or $\geq 16 \mathrm{~kg}$ in women) muscle strength and pathological (SARC-F $\geq 4$ ) or normal (SARC-F $<4$ ) values of the sarcopenia screening tool. Comparisons were made with the chi-squared test for categorical variables, and with the MannWhitney $U$ test, for continuous variables. Cut-off values for muscle performance and strength were chosen, according to the European Working Group on Sarcopenia Guidelines (1) and to literature data (36).

We assessed the correlations between muscle ultrasound characteristics (muscle thickness, pennation angle and muscle stiffness), measures of muscle function (SPPB and Hand Grip Strength), nutritional status (MNA-SF), age, SARC-F, inflammatory indexes [highest $\mathrm{C}$ Reactive Protein (CRP) and number of days with CRP above the upper normal limit, highest ferritin during hospital stay, highest white blood cells during hospital stay (WBC)] and length of hospital stay through Spearman correlations.

Probable sarcopenia was defined as a reduced muscle strength at the hand grip test, accordingly to the European Working Group on Sarcopenia Guidelines (1). Binary logistic regression analyses were used, to assess the association between muscle ultrasound parameters, and probable sarcopenia. Unadjusted and stepwise adjusted models were performed. Collinearity tests were run before performing the adjusted model; collinear variables were excluded from the multivariable model, and just one proxy of the severity of SARS-CoV-2 infection (NonInvasive Mechanical Ventilation) and of clinical complexity before hospital admission (number of chronic therapies) were inserted in the model, in addition to the significant predictors at the univariable analyses.

Finally, we performed ROC analyses, to identify optimal cutoff values of muscle ultrasound characteristics (thickness, stiffness and pennation angle) associated with probable sarcopenia. Reduced muscle strength (defined as a Hand Grip Strength $<27 \mathrm{~kg}$ in men or $<16 \mathrm{~kg}$ in women) was used as state variable. The Area under the curve (AUC) was calculated as a summary of diagnostic accuracy. Maximum value of the Youden's index was used for selecting the optimum cutoff points.

All statistical analyses were performed with SPSS version 25.0 (SPSS Inc., Chicago, IL, USA).

\section{RESULTS}

Two hundred and fifty-nine patients seen at a dedicated postCOVID-19 outpatient clinic, were included in the study. Table 1 shows the main baseline characteristics of the study population. Table 2 illustrates the main characteristics of their COVID-19 hospitalization. Table 3 provides information on patients' muscle and nutritional characteristics, one month after hospital discharge.

The comparisons between patients with pathological versus normal values of muscle function and of SARC-F are shown in Tables 1-3. Many patients presented an overlap of pathological tests as illustrated by the Venn Diagram in Figure 1.

The patients with an impaired muscle function or with a pathological SARC-F score were older and more often females, compared to individuals with a normal muscle function. They had a lower weight and a higher burden of chronic therapies, both before hospital admission and one month after hospital discharge. Furthermore, their muscle stiffness was higher. Pennation angle and weight loss during hospital stay did not differ. Muscle thickness was significantly lower in patients with reduced versus those with normal muscle strength (1.6 versus $1.73 \mathrm{~cm}, \mathrm{p}=0.02$ ). This finding was confirmed in patients with reduced versus those with normal muscle performance (1.3 
TABLE 1 | Baseline characteristics of the study population and their comparison among groups with pathologic and normal values of a sarcopenia screening tool, muscle strength and performance.

\begin{tabular}{|c|c|c|c|c|c|c|c|c|c|c|}
\hline & \multirow{2}{*}{$\begin{array}{c}\text { Total } \\
\text { sample } \\
\text { (N = 259) }\end{array}$} & \multicolumn{2}{|c|}{ SARC-F } & \multirow[t]{2}{*}{$\mathbf{p}$} & \multicolumn{2}{|c|}{ GRIP STRENGTH } & \multirow[t]{2}{*}{$\mathbf{p}$} & \multicolumn{2}{|c|}{ SPPB } & \multirow[t]{2}{*}{$\mathbf{p}$} \\
\hline & & $\geq 4(\mathrm{~N}=32)$ & $<4(\mathrm{~N}=222)$ & & Low * $(N=121)$ & Normal (N = 134) & & $\leq 8(N=14)$ & $>8(\mathrm{~N}=238)$ & \\
\hline Age & $\begin{array}{c}67 \text { (IQR } 56- \\
75)\end{array}$ & $\begin{array}{l}74.5(\mathrm{IQR} \\
62.5-80)\end{array}$ & $\begin{array}{c}66 \text { (IQR } 55.75 \\
-74)\end{array}$ & 0.01 & 73 (IQR $63-79)$ & $\begin{array}{c}61.0(\text { IQR } 52.0- \\
69.0)\end{array}$ & $\begin{array}{c}< \\
0.001\end{array}$ & $\begin{array}{c}80.5 \text { (IQR } 74- \\
83.75)\end{array}$ & $\begin{array}{c}66.0(\text { IQR } \\
55.0-73.0)\end{array}$ & $\begin{array}{c}< \\
0.001\end{array}$ \\
\hline Males & 155 (59.8\%) & $5(15.6 \%)$ & 147 (66.2\%) & $\begin{array}{c}< \\
0.001\end{array}$ & $68(56.2 \%)$ & $84(62.7 \%)$ & 0.3 & $5(35.7 \%)$ & $148(62.2 \%)$ & 0.049 \\
\hline \multicolumn{11}{|l|}{ Smoke } \\
\hline Never & 135 (52.1\%) & 19 (59.4\%) & 112 (50.7\%) & 0.47 & $61(50.4 \%)$ & 72 (53.7\%) & 0.21 & 7 (50\%) & $124(52 \%)$ & 0.88 \\
\hline Previous & 97 (37.5\%) & $9(28.1 \%)$ & 87 (39.2\%) & & $51(42.1 \%)$ & 44 (32.8\%) & & $5(35.7 \%)$ & 89 (37.4\%) & \\
\hline Active & 26 (10\%) & $4(12.5 \%)$ & 22 (9.9\%) & & $9(7.4 \%)$ & 17 (12.7\%) & & $2(14.3 \%)$ & $24(10.1 \%)$ & \\
\hline Alcohol abuse & $6(2.3 \%)$ & $0(0 \%)$ & $6(2.7 \%)$ & 0.02 & $5(4.1 \%)$ & $1(0.7 \%)$ & 0.11 & $0(0 \%)$ & $6(2.5 \%)$ & 0.81 \\
\hline $\begin{array}{l}\text { Bedridden before } \\
\text { hospital admission }\end{array}$ & $2(0.8 \%)$ & $1(3.1 \%)$ & $1(0.5 \%)$ & 0.11 & $2(1.7 \%)$ & $0(0 \%)$ & 0.13 & $0(0 \%)$ & $1(0.4 \%)$ & 0.81 \\
\hline $\begin{array}{l}\text { Weight }(\mathrm{kg}) \text { before } \\
\text { hospital admission }\end{array}$ & $\begin{array}{c}79 \text { (IQR } 69- \\
91)\end{array}$ & $\begin{array}{l}70(\mathrm{IQR} \\
62.25- \\
80.75)\end{array}$ & $\begin{array}{c}81.0 \text { (IQR } 70- \\
92)\end{array}$ & 0.005 & 75 (IQR 65 - 90) & $83.0(\mathrm{SD} \pm 15.65)$ & 0.008 & $\begin{array}{c}70 \text { (IQR } 66.75 \\
-77.75)\end{array}$ & $\begin{array}{c}80.0(\mathrm{IQR} \\
69.5-92.0)\end{array}$ & 0.04 \\
\hline $\begin{array}{l}\text { BMl (kg/m2) before } \\
\text { hospital admission }\end{array}$ & $\begin{array}{l}28(\mathrm{IQR} \\
24.87- \\
31.01)\end{array}$ & $\begin{array}{c}28(\mathrm{IQR} \\
23.94- \\
31.54)\end{array}$ & $\begin{array}{c}27.7(\mathrm{IQR} \\
24.87-30.82)\end{array}$ & 0.81 & $\begin{array}{c}27.3 \text { (IQR } 24.56- \\
31.83)\end{array}$ & $\begin{array}{c}28.1 \text { (IQR 25.12 - } \\
\text { 30.32) }\end{array}$ & 0.63 & $\begin{array}{c}27.1(\mathrm{IQR} \\
23.65-29.38)\end{array}$ & $\begin{array}{c}28(\mathrm{IQR} \\
24.99- \\
31.14)\end{array}$ & 0.29 \\
\hline $\begin{array}{l}\text { Chronic therapies at } \\
\text { hospital admission }\end{array}$ & $3(\operatorname{IQR} 1$ - 5) & $\begin{array}{c}5.5 \text { (IQR } 3.25 \\
\quad-7.75)\end{array}$ & $2(\operatorname{lQR} 1-5)$ & $\begin{array}{c}< \\
0.001\end{array}$ & $4.53(\mathrm{SD} \pm 3.46)$ & $2(\operatorname{IQR} 0-4)$ & $\begin{array}{c}< \\
0.001\end{array}$ & $\begin{array}{c}6 \text { (IQR } 1.75- \\
9)\end{array}$ & $3(\operatorname{QQR} 1$ - 5) & 0.02 \\
\hline Chronic steroid use & $10(3.9 \%)$ & $3(9.4 \%)$ & $7(3.2 \%)$ & 0.07 & $4(3.3 \%)$ & $6(4.5 \%)$ & 0.67 & $0(0 \%)$ & $10(4.2 \%)$ & 0.43 \\
\hline Hypertension & $132(51 \%)$ & $17(53.1 \%)$ & $112(50.5 \%)$ & 0.78 & $70(57.9 \%)$ & $59(44 \%)$ & 0.04 & $11(78.6 \%)$ & $116(48.7 \%)$ & 0.03 \\
\hline Diabetes & $53(20.5 \%)$ & 7 (21.9\%) & $45(20.3 \%)$ & 0.83 & $33(27.3 \%)$ & 19 (14.2\%) & 0.008 & $5(35.7 \%)$ & 46 (19.3\%) & 0.14 \\
\hline CKD & $20(7.7 \%)$ & $3(9.7 \%)$ & $16(7.3 \%)$ & 0.68 & $13(11 \%)$ & $6(4.4 \%)$ & 0.10 & $3(21.4 \%)$ & $16(6.8 \%)$ & 0.12 \\
\hline Arrhythmia & $30(11.6 \%)$ & $5(15.6 \%)$ & $24(10.8 \%)$ & 0.42 & $18(14.9 \%)$ & $11(8.2 \%)$ & 0.09 & $5(35.7 \%)$ & 23 (9.7\%) & 0.003 \\
\hline Ischemic heart disease & 28 (10.8\%) & $3(9.4 \%)$ & 25 (11.3\%) & 0.75 & 18 (14.9\%) & $10(7.5 \%)$ & 0.05 & $4(28.6 \%)$ & 24 (10.1\%) & 0.03 \\
\hline Stroke/TIA & $12(4.6 \%)$ & $3(9.4 \%)$ & $9(4.1 \%)$ & 0.18 & $9(7.4 \%)$ & $3(2.2 \%)$ & 0.047 & $2(14.3 \%)$ & $10(4.2 \%)$ & 0.08 \\
\hline $\begin{array}{l}\text { Peripheral vascular } \\
\text { disease }\end{array}$ & $28(10.8 \%)$ & $3(9.4 \%)$ & $25(11.3 \%)$ & 0.75 & $16(13.2 \%)$ & 12 (9\%) & 0.26 & $1(7.1 \%)$ & $27(11.3 \%)$ & 0.63 \\
\hline COPD & $15(5.8 \%)$ & $2(6.3 \%)$ & 13 (5.9\%) & 0.93 & $11(9.1 \%)$ & $4(3 \%)$ & 0.04 & $3(21.4 \%)$ & $12(5 \%)$ & 0.01 \\
\hline Asthma & $9(3.5 \%)$ & $3(9.4 \%)$ & $6(2.7 \%)$ & 0.05 & $4(3.3 \%)$ & $5(3.7 \%)$ & 0.87 & $0(0 \%)$ & $9(3.8 \%)$ & 0.46 \\
\hline $\begin{array}{l}\text { Other respiratory } \\
\text { disease }\end{array}$ & $17(6.6 \%)$ & $2(6.3 \%)$ & $14(6.3 \%)$ & 0.98 & $9(7.4 \%)$ & $7(5.2 \%)$ & 0.44 & $1(7.1 \%)$ & $15(6.3 \%)$ & 0.90 \\
\hline Chronic anemia & $9(3.5 \%)$ & $3(9.4 \%)$ & $6(2.7 \%)$ & 0.05 & $6(5 \%)$ & $3(2.2 \%)$ & 0.23 & $2(14.3 \%)$ & 7 (2.9\%) & 0.03 \\
\hline Osteoporosis & $12(4.6 \%)$ & 5 (15.6\%) & $5(2.3 \%)$ & $\begin{array}{c}< \\
0.001\end{array}$ & $8(6.6 \%)$ & $4(3 \%)$ & 0.16 & $1(7.1 \%)$ & $9(3.8 \%)$ & 0.53 \\
\hline Arthrosis & 17 (6.6\%) & $3(9.4 \%)$ & 13 (5.9\%) & 0.44 & $9(7.4 \%)$ & $8(6 \%)$ & 0.62 & $1(7.1 \%)$ & 14 (5.9\%) & 0.85 \\
\hline Rheumatic disease & $11(4.2 \%)$ & $3(9.4 \%)$ & $8(3.6 \%)$ & 0.13 & $5(4.1 \%)$ & $6(4.5 \%)$ & 0.91 & $1(7.1 \%)$ & $10(4.2 \%)$ & 0.60 \\
\hline Dementia & $3(1.2 \%)$ & $2(6.3 \%)$ & $1(0.5 \%)$ & 0.005 & $2(1.7 \%)$ & $1(0.7 \%)$ & 0.49 & $1(7.1 \%)$ & $1(0.4 \%)$ & 0.006 \\
\hline $\begin{array}{l}\text { Other neurologic } \\
\text { diseases }\end{array}$ & $22(8.5 \%)$ & 5 (15.6\%) & $17(7.7 \%)$ & 0.13 & 16 (13.2\%) & $4(3 \%)$ & 0.01 & $4(28.6 \%)$ & $15(6.3 \%)$ & 0.002 \\
\hline Psychiatric disease & 32 (12.4\%) & 7 (21.9\%) & 24 (10.8\%) & 0.07 & $20(16.5 \%)$ & $12(9 \%)$ & 0.06 & $4(28.6 \%)$ & 26 (10.9\%) & 0.048 \\
\hline Vitamin D deficit & $15(5.8 \%)$ & $5(15.6 \%)$ & $9(4.1 \%)$ & 0.008 & $11(9.1 \%)$ & $4(3 \%)$ & 0.03 & $1(7.1 \%)$ & 12 (5\%) & 0.73 \\
\hline Active neoplasm & $18(6.9 \%)$ & $2(6.3 \%)$ & $16(7.2 \%)$ & 0.84 & $10(8.3 \%)$ & $8(6 \%)$ & 0.45 & $2(14.3 \%)$ & $16(6.7 \%)$ & 0.29 \\
\hline
\end{tabular}

*Hand grip strength < $27 \mathrm{~kg}$ in men; < $16 \mathrm{~kg}$ in woman.

SARC-F,Screening tool for sarcopenia; SPPB, Short Physical Performance Battery; IQR, Inter Quartile Range; BMI, Body Mass Index; CKD, Chronic Kidney Disease; TIA, Transient Ischemic attack; COPD, Chronic Obstructive Pulmonary Disease. Bold = statistically significant $(p<0.05)$.

versus $1.71 \mathrm{~cm}, \mathrm{p}=0.01)$. Muscle stiffness was higher in patients with reduced muscle strength compared to patients with normal muscle strength ( 87 versus $76.3, \mathrm{p}=0.004$ ). Also in patients with reduced muscle performance muscle stiffness was higher compared to patients with normal muscle performance (104.9 versus $81.07, \mathrm{p}=0.04)$. Figure 2 illustrates the muscle US images of a patient with reduced muscle strength (i.e. probable sarcopenia) and of a patient with normal muscle strength.

Osteoporosis $(\mathrm{p}<0.001)$, dementia $(\mathrm{p}=0.005)$ and vitamin $\mathrm{D}$ deficiency ( $p=0.008)$ were more prevalent in people with a high SARC-F score, as compared to those with normal values.
Moreover, the patients with a high SARC-F score had higher serum ferritin during hospital stay $(p=0.03)$, and worse nutritional status, one month after hospital discharge (MNASF: 8 versus $9, \mathrm{p}=0.007)$.

The patients with probable sarcopenia were more frequently affected by hypertension $(p=0.04)$, diabetes $(p=0.008)$, stroke or previous transient ischemic attack $(p=0.047)$, chronic obstructive pulmonary disease (COPD) $(\mathrm{p}=0.04)$, neurological diseases, different from dementia $(p=0.01)$ and vitamin $D$ deficiency ( $p=0.03$ ). Moreover, admission to the Intensive Care Unit was more frequent $(\mathrm{p}=0.04)$, and WBC $(\mathrm{p}=0.01)$ 
TABLE 2 | Main characteristics of the COVID-19 hospitalization of the study population and their comparison among groups with pathologic and normal values of a sarcopenia screening tool, muscle strength and performance.

\begin{tabular}{|c|c|c|c|c|c|c|c|c|c|c|}
\hline & \multirow{2}{*}{$\begin{array}{c}\text { Total } \\
\text { sample } \\
(\mathrm{N}=259)\end{array}$} & \multicolumn{2}{|c|}{ SARC-F } & \multirow[t]{2}{*}{$\mathbf{p}$} & \multicolumn{2}{|c|}{ GRIP STRENGTH } & \multirow[t]{2}{*}{$\mathbf{p}$} & \multicolumn{2}{|c|}{ SPPB } & \multirow[t]{2}{*}{$\mathbf{p}$} \\
\hline & & $\geq 4(N=32)$ & $<4(\mathrm{~N}=222)$ & & $\begin{array}{l}\text { Reduced * } \\
(N=121)\end{array}$ & $\begin{array}{l}\text { Normal } \\
(\mathrm{N}=134)\end{array}$ & & $\leq 8(N=14)$ & $>8(\mathrm{~N}=238)$ & \\
\hline $\begin{array}{l}\text { Weight loss during hospital } \\
\text { stay }(\mathrm{kg})\end{array}$ & $\begin{array}{c}5(\mathrm{IQR} 3- \\
7.1)\end{array}$ & $\begin{array}{c}4.25(\mathrm{IQR} 2- \\
5)\end{array}$ & $\begin{array}{c}5.0(\mathrm{IQR} 3.0- \\
8.0)\end{array}$ & 0.22 & $5($ IQR $3-9)$ & $\begin{array}{c}5.0(\text { IQR } 2.0- \\
7.0)\end{array}$ & 0.2 & $\begin{array}{c}4.5 \text { (IQR } 2.75- \\
5)\end{array}$ & $\begin{array}{c}5 \text { (IQR } 3.0- \\
7.65)\end{array}$ & 0.56 \\
\hline $\begin{array}{l}\text { Length of hospital stay } \\
\text { (days) }\end{array}$ & $\begin{array}{c}14 \text { (IQR } 9- \\
21)\end{array}$ & $\begin{array}{c}18.5(\text { IQR } 9- \\
31.25)\end{array}$ & $\begin{array}{c}13.0(\text { IQR } 9 \text { - } \\
20.25)\end{array}$ & 0.19 & $\begin{array}{c}16(\mathrm{IQR} 10- \\
28.5)\end{array}$ & $\begin{array}{c}11.0 \text { (IQR } 8.0- \\
17.0)\end{array}$ & $\begin{array}{c}< \\
0.001\end{array}$ & $\begin{array}{c}18.5 \text { (IQR } 9.75 \\
-35.25)\end{array}$ & $\begin{array}{c}13.5 \text { (IQR } 9 \text { - } \\
21)\end{array}$ & 0.13 \\
\hline ICU stay & $22(8.5 \%)$ & $3(9.4 \%)$ & $19(8.6 \%)$ & 0.88 & 15 (12.4\%) & $7(5.2 \%)$ & 0.04 & $3(21.4 \%)$ & $19(8 \%)$ & 0.08 \\
\hline Length of ICU stay & $\begin{array}{c}14 \text { (IQR } 6.5- \\
29.5)\end{array}$ & $\begin{array}{c}18(\mathrm{IQR} 18 \text { - } \\
18)\end{array}$ & $\begin{array}{c}10.0 \text { (IQR } 5.75 \\
-32.50)\end{array}$ & 0.76 & 17 (IQR 8 -33) & $\begin{array}{c}9.0(\text { IQR } 5.0- \\
19.0)\end{array}$ & 0.08 & $\begin{array}{c}17 \text { (IQR } 17- \\
17)\end{array}$ & $\begin{array}{c}11.0 \text { (IQR } 6.0- \\
32.0)\end{array}$ & 0.96 \\
\hline NIV & 67 (25.9\%) & $7(21.9 \%)$ & $59(26.6 \%)$ & 0.57 & 40 (33.1\%) & $26(19.4 \%)$ & 0.01 & $2(14.3 \%)$ & $64(26.9 \%)$ & 0.30 \\
\hline $\begin{array}{l}\text { Steroid use during hospital } \\
\text { stay }\end{array}$ & $242(93.5 \%)$ & $29(90.6 \%)$ & $208(93.7 \%)$ & 0.52 & $113(93.4 \%)$ & $125(93.3 \%)$ & 0.99 & $14(100 \%)$ & $222(93.3 \%)$ & 0.32 \\
\hline Anakinra use & 36 (13.9\%) & $1(3.1 \%)$ & 33 (14.9\%) & 0.07 & $16(13.2 \%)$ & $18(13.4 \%)$ & 0.86 & $1(7.1 \%)$ & 34 (14.3\%) & 0.45 \\
\hline $\begin{array}{l}\text { Highest CRP during } \\
\text { hospital stay (mg/l) }\end{array}$ & $\begin{array}{c}70.7(\mathrm{IQR} \\
40.5-119.2)\end{array}$ & $\begin{array}{c}68.3(\mathrm{IQR} \\
42.0-125.87)\end{array}$ & $\begin{array}{c}68.5(\mathrm{IQR} \\
39.95- \\
118.85)\end{array}$ & 0.70 & $\begin{array}{c}81.1(\mathrm{IQR} \\
40.75- \\
131.35)\end{array}$ & $\begin{array}{c}63.0(\mathrm{IQR} \\
37.52- \\
103.35)\end{array}$ & 0.06 & $\begin{array}{c}81.25 \text { (IQR } \\
55.10- \\
169.65)\end{array}$ & $\begin{array}{c}69.8(\mathrm{IQR} \\
40.67- \\
117.52)\end{array}$ & 0.30 \\
\hline $\begin{array}{l}\text { Days with CRP above the } \\
\text { normal limit }\end{array}$ & $\begin{array}{c}8(\mathrm{IQR} 6- \\
13)\end{array}$ & $\begin{array}{c}10(\text { IQR } 6.0- \\
19.5)\end{array}$ & $8.0($ IQR 6 - 12) & 0.13 & 10 (IQR 6 - 20) & $\begin{array}{c}8.0(\text { IQR } 5.0- \\
10.0)\end{array}$ & $\begin{array}{c}< \\
0.001\end{array}$ & $\begin{array}{c}11 \text { (IQR } 7- \\
25.75)\end{array}$ & $\begin{array}{c}8.0 \text { (IQR } 6.0- \\
12.75)\end{array}$ & 0.03 \\
\hline $\begin{array}{l}\text { Highest ferritin during } \\
\text { hospital stay (ng/ml) }\end{array}$ & $\begin{array}{c}943 \text { (IQR } \\
516-1522)\end{array}$ & $\begin{array}{c}639 \text { (IQR } \\
291.0- \\
1312.0)\end{array}$ & $\begin{array}{c}968.0 \text { (IQR } \\
592.0- \\
1538.0)\end{array}$ & 0.03 & $\begin{array}{c}934 \text { (IQR } \\
488.75- \\
1654.0)\end{array}$ & $\begin{array}{c}919.0 \text { (IQR } \\
520.0- \\
1370.0)\end{array}$ & 0.50 & $\begin{array}{c}556 \text { (IQR } 217 \text { - } \\
\text { 2096) }\end{array}$ & $\begin{array}{c}960.0 \text { (IQR } \\
549.0- \\
1522.0)\end{array}$ & 0.31 \\
\hline $\begin{array}{l}\text { Highest WBC during hospital } \\
\text { stay }\left(10^{\wedge} 3 \text { cells } / \mathrm{mmc}\right)\end{array}$ & $\begin{array}{c}9.9 \text { (IQR } 8.0 \\
-12.9)\end{array}$ & $\begin{array}{c}9.3(\mathrm{IQR} 7.55 \\
-12.80)\end{array}$ & $\begin{array}{c}10.2 \text { (IQR } 8.1- \\
12.9)\end{array}$ & 0.57 & $\begin{aligned} 10.7 & \text { (IQR } 8.75 \\
& -13.25)\end{aligned}$ & $\begin{array}{c}9.5(\mathrm{IQR} 7.4- \\
12.4)\end{array}$ & 0.01 & $\begin{array}{c}9.35 \text { (IQR } 8.77 \\
-12.65)\end{array}$ & $\begin{array}{c}10.1 \text { (IQR } 8.0- \\
12.9)\end{array}$ & 0.88 \\
\hline
\end{tabular}

*Hand grip strength < $27 \mathrm{~kg}$ in men; < $16 \mathrm{~kg}$ in woman.

SARC-F,Screening tool for sarcopenia; SPPB, Short Physical Performance Battery; IQR, Inter Quartile Range; ICU, Intensive Care Unit; NIV, Non Invasive Ventilation; CRP, C Reactive Protein; WBC, White Blood Cells. Bold = statistically significant $(p<0.05)$.

TABLE 3 | Muscle and nutritional characteristics one month after hospital discharge of the study population and their comparison among groups with pathologic and normal values of a sarcopenia screening tool, muscle strength and performance.

\begin{tabular}{|c|c|c|c|c|c|c|c|c|c|c|}
\hline & \multirow{2}{*}{$\begin{array}{l}\text { Total sample } \\
\qquad(\mathrm{N}=\mathbf{2 5 9})\end{array}$} & \multicolumn{2}{|c|}{ SARC-F } & \multirow[t]{2}{*}{$\mathbf{p}$} & \multicolumn{2}{|c|}{ GRIP STRENGTH } & \multirow[t]{2}{*}{$\mathbf{p}$} & \multicolumn{2}{|c|}{ SPPB } & \multirow[t]{2}{*}{ p } \\
\hline & & $\geq 4(\mathrm{~N}=32)$ & $<4(\mathrm{~N}=222)$ & & $\begin{array}{l}\text { Reduced * } \\
(N=121)\end{array}$ & $\begin{array}{l}\text { Normal } \\
(\mathrm{N}=134)\end{array}$ & & $\leq 8(N=14)$ & $>8(N$ & \\
\hline $\begin{array}{l}\text { Weight }(\mathrm{kg}) 1 \text { month after } \\
\text { hospital discharge }\end{array}$ & $\begin{array}{c}77(\mathrm{IQR} 66- \\
87)\end{array}$ & $\begin{array}{c}69.5(\mathrm{IQR} \\
59.2-77.7)\end{array}$ & $\begin{array}{c}78.0 \text { (IQR } 67- \\
88)\end{array}$ & 0.004 & $\begin{array}{c}73(\mathrm{IQR} 62- \\
84)\end{array}$ & $\begin{array}{c}80.0(\mathrm{IQR} \\
70.0-88.0)\end{array}$ & $\begin{array}{c}< \\
0.001\end{array}$ & $\begin{array}{l}67 \text { (IQR } 59- \\
77.25)\end{array}$ & $\begin{array}{c}77.0(\mathrm{IQR} \\
67.0-88.0)\end{array}$ & 0.02 \\
\hline $\begin{array}{l}\text { BMI }(\mathrm{kg} / \mathrm{m} 2) 1 \text { month hospital } \\
\text { discharge }\end{array}$ & $\begin{array}{l}27 \text { (IQR } 24.22 \\
-29.47)\end{array}$ & $\begin{array}{c}26.75 \text { (IQR } \\
23.10- \\
30.27)\end{array}$ & $\begin{array}{c}27 \text { (IQR } 24.34 \\
-29.41)\end{array}$ & 0.57 & $\begin{array}{c}26.6 \text { (IQR } \\
23.42- \\
29.34)\end{array}$ & $\begin{array}{c}27.1(\mathrm{IQR} \\
24.74- \\
29.71)\end{array}$ & 0.14 & $\begin{array}{c}26.7(\mathrm{IQR} \\
22.10- \\
28.39)\end{array}$ & $\begin{array}{c}27.1(\mathrm{IQR} \\
24.43- \\
29.69)\end{array}$ & 0.22 \\
\hline $\begin{array}{l}\text { Chronic therapies } 1 \text { month } \\
\text { after hospital discharge }\end{array}$ & $3(\operatorname{IQR} 1-6)$ & $6(\operatorname{IQR} 4-11)$ & $\begin{array}{c}3.0(\mathrm{IQR} 1.0- \\
6.0)\end{array}$ & $\begin{array}{c}< \\
0.001\end{array}$ & $5(\mathrm{IQR} 3-7)$ & $\begin{array}{c}2.0(\mathrm{IQR} 1.0- \\
5.0)\end{array}$ & $\begin{array}{c}< \\
0.001\end{array}$ & $\begin{array}{c}8 \text { (IQR } 3.75- \\
12.25)\end{array}$ & $\begin{array}{c}3.0(\operatorname{IQR} 1.0- \\
6.0)\end{array}$ & 0.002 \\
\hline $\begin{array}{l}\text { Muscle thickness }(\mathrm{cm}) 1 \\
\text { month after hospital discharge }\end{array}$ & $\begin{array}{c}1.7 \text { (IQR } 1.44 \\
-1.93)\end{array}$ & $\begin{array}{c}1.6(\operatorname{IQR} 1.33 \\
-1.80)\end{array}$ & $\begin{array}{l}1.7 \text { (IQR } 1.45 \\
-1.95)\end{array}$ & 0.17 & $\begin{array}{c}1.6(\operatorname{IQR} 1.36 \\
-1.87)\end{array}$ & $\begin{array}{c}1.73(\mathrm{IQR} \\
1.52-1.99)\end{array}$ & 0.02 & $\begin{array}{c}1.3(\mathrm{IQR} 1.14 \\
-1.65)\end{array}$ & $\begin{array}{c}1.71(\mathrm{IQR} \\
1.47-1.96)\end{array}$ & 0.01 \\
\hline $\begin{array}{l}\text { Pennation angle }\left(^{\circ}\right) 1 \text { month } \\
\text { after hospital discharge }\end{array}$ & $\begin{array}{c}22.4(\mathrm{IQR} \\
19.70-26.0)\end{array}$ & $\begin{array}{c}22(\text { IQR } 18.0- \\
24.8)\end{array}$ & $\begin{array}{c}22.9 \text { (IQR } \\
19.80- \\
26.20)\end{array}$ & 0.20 & $\begin{array}{c}22(\text { IQR 19.12 } \\
-26.0)\end{array}$ & $\begin{array}{c}23.0(\mathrm{IQR} \\
20.37- \\
26.55)\end{array}$ & 0.15 & $\begin{array}{c}20.0(\mathrm{IQR} \\
16.75- \\
23.90)\end{array}$ & $\begin{array}{c}22.8(\mathrm{IQR} \\
19.85- \\
26.10)\end{array}$ & 0.06 \\
\hline $\begin{array}{l}\text { Muscle stiffness } 1 \text { month after } \\
\text { hospital discharge }^{\star \star}\end{array}$ & $\begin{array}{c}81.43 \text { (IQR } \\
65.02- \\
97.32)\end{array}$ & $\begin{array}{c}103.0 \text { (IQR } \\
94.73- \\
111.0)\end{array}$ & $\begin{array}{c}79.55(\mathrm{IQR} \\
62.57- \\
93.98)\end{array}$ & $\begin{array}{c}< \\
0.001\end{array}$ & $\begin{array}{c}87.0(\mathrm{IQR} \\
74.82- \\
103.10)\end{array}$ & $\begin{array}{c}76.3(\mathrm{SD} \pm \\
22.72)\end{array}$ & 0.004 & $\begin{array}{c}104.9(\text { IQR } \\
82.81-110)\end{array}$ & $\begin{array}{c}81.07 \text { (IQR } \\
64.03- \\
97.23)\end{array}$ & 0.04 \\
\hline $\begin{array}{l}\text { MNA-SF } 1 \text { month after hospital } \\
\text { discharge }\end{array}$ & $9(\operatorname{QQR} 8-11)$ & $\begin{array}{c}8 \text { (IQR } 7.0- \\
10.0)\end{array}$ & $9(\operatorname{IQR} 8-11)$ & 0.007 & $\begin{array}{c}9.0(\mathrm{IQR} 7- \\
11)\end{array}$ & $\begin{array}{c}9.0(\text { IQR } 8.0- \\
11.0)\end{array}$ & 0.08 & $\begin{array}{c}8.5(\text { IQR } 6- \\
10)\end{array}$ & $\begin{array}{c}9.0(\text { IQR } 8.0- \\
11.0)\end{array}$ & 0.07 \\
\hline $\begin{array}{l}\text { SPPB } 1 \text { month after hospital } \\
\text { discharge }\end{array}$ & $\begin{array}{c}12(\mathrm{IQR} 12- \\
12)\end{array}$ & $\begin{array}{l}11(\text { IQR } 8.0- \\
12.0)\end{array}$ & $\begin{array}{c}12(\mathrm{IQR} 12- \\
12)\end{array}$ & $\begin{array}{c}< \\
0.001\end{array}$ & $\begin{array}{l}12(\text { IQR } 10.25 \\
-12)\end{array}$ & $\begin{array}{c}12.0(\mathrm{IQR} \\
12.0-12.0)\end{array}$ & $\begin{array}{c}< \\
0.001\end{array}$ & $\begin{array}{c}7.5(\operatorname{IQR} 5- \\
8)\end{array}$ & $\begin{array}{c}12.0(\mathrm{IQR} \\
12.0-12.0)\end{array}$ & $\begin{array}{c}< \\
0.001\end{array}$ \\
\hline Hand Grip Strength (kg) 1 & $21.7(\mathrm{IQR}$ & $14.5(\mathrm{IQR}$ & $24.3(\mathrm{IQR}$ & $<$ & $15.9(\mathrm{IQR}$ & $29.25(\mathrm{IQR}$ & $<$ & $12.2(\mathrm{IQR}$ & $23.2(\mathrm{IQR}$ & $<$ \\
\hline month after hospital discharge & $15.95-30.0)$ & $11.35-16.1)$ & $17.5-31.3)$ & 0.001 & $12.8-22.25)$ & $\begin{array}{l}19.63- \\
33.97)\end{array}$ & 0.001 & $\begin{array}{l}10.95- \\
15.90)\end{array}$ & $\begin{array}{l}16.72- \\
30.70)\end{array}$ & 0.001 \\
\hline $\begin{array}{l}\text { SARCF } 1 \text { month after hospital } \\
\text { discharge }\end{array}$ & $1($ IQR 0 - 2) & $\begin{array}{c}4.5(\mathrm{IQR} 4.0- \\
5.0)\end{array}$ & $1($ IQR 0 - 2) & $\begin{array}{c}< \\
0.001\end{array}$ & $2(\operatorname{IQR} 1$ - 3) & $\begin{array}{c}0.0 \text { (IQR } 0.0 \text { - } \\
1.0)\end{array}$ & $\begin{array}{c}< \\
0.001\end{array}$ & $\begin{array}{c}4 \text { (IQR } 2.75- \\
5)\end{array}$ & $\begin{array}{c}1.0 \text { (IQR } 0.0- \\
2.0)\end{array}$ & $\begin{array}{c}< \\
0.001\end{array}$ \\
\hline
\end{tabular}

*Hand grip strength < $27 \mathrm{~kg}$ in men; < $16 \mathrm{~kg}$ in woman.

**data on muscle stiffness were available for only 152 patients.

SARC-F, Screening tool for sarcopenia; SPPB, Short Physical Performance Battery; IQR, Inter Quartile Range; BMI, Body Mass Index; MNA-SF, Mini Nutritional Assessment Short Form. Bold = statistically significant $(p<0.05)$. 


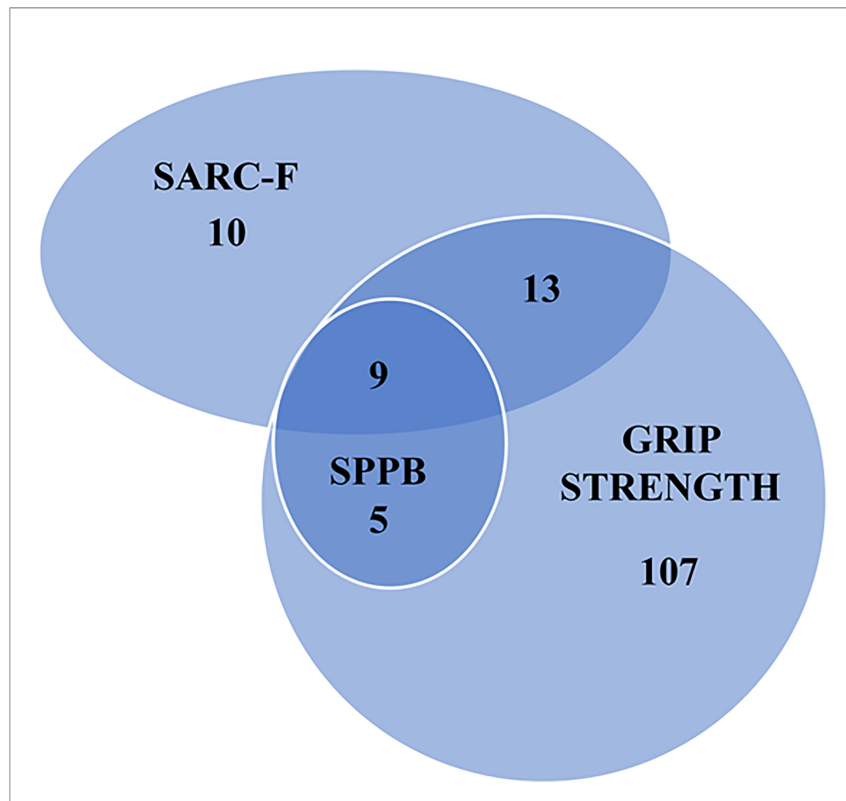

FIGURE 1 | Number of people with a pathologic value of a sarcopenia screening tool, hand grip strength and muscle performance test. SARC-F, Screening tool for sarcopenia; SPPB, Short Physical Performance Battery.

and days of $\mathrm{C}$ Reactive Protein (CRP) above the upper normal limit during hospitalization $(\mathrm{p}<0.001)$ were higher in patients with probable sarcopenia.

The patients with a pathological muscle performance were more comorbid than patients with normal muscle performance. In particular, they were more frequently affected by hypertension $(\mathrm{p}=0.03)$, ischemic heart disease $(\mathrm{p}=0.03)$, arrhythmia $(\mathrm{p}=$
0.003), COPD $(\mathrm{p}=0.01)$, chronic anaemia $(\mathrm{p}=0.03)$, dementia $(\mathrm{p}=0.006)$ and other neurological $(\mathrm{p}=0.002)$ and psychiatric diseases $(\mathrm{p}=0.048)$. In addition, during hospitalization, they had CRP levels above the upper normal limit, for a longer time $(\mathrm{p}=0.03)$.

Table 4 shows the results of the Spearman correlations among muscle ultrasound characteristics, and the measures of muscle function and nutritional status, age, inflammatory indexes, and length of hospital stay.

We detected significant direct correlations, between muscle thickness and pennation angle ( $\mathrm{p} 0.46, \mathrm{p}<0.001)$, MNA-SF ( $\mathrm{p} 0.16, \mathrm{p}=0.01)$, grip strength $(\mathrm{p} 0.32, \mathrm{p}<0.001)$, SPPB ( $\mathrm{p} 0.2$, $\mathrm{p}=0.001)$ and ferritin $(\mathrm{p} 0.16, \mathrm{p}=0.01)$ and an inverse correlation of muscle thickness with SARC-F ( $\mathrm{p}-0.23, \mathrm{p}<$ $0.001)$ and with age $(\mathrm{p}-0.35, \mathrm{p}<0.001)$.

Pennation angle had a direct correlation with nutritional status ( $\mathrm{p} 0.17, \mathrm{p}=0.008$ ), and with muscle function (Hand Grip Strength: $\mathrm{p}$ $0.16, \mathrm{p}=0.01 ; \mathrm{SPPB}: \mathrm{p} 0.13, \mathrm{p}=0.03)$ and an inverse correlation with SARC-F $(\mathrm{p}-0.19, \mathrm{p}=0.005)$ and with age $(\mathrm{p}-0.21, \mathrm{p}=0.001)$. Instead, muscle stiffness showed a direct correlation with age ( $\mathrm{p}$ $0.22, \mathrm{p}=0.007)$ and with SARC-F ( $0.3, \mathrm{p}<0.001)$. Muscle stiffness had an inverse correlation with grip strength ( $\mathrm{p}-0.26, \mathrm{p}=0.001)$, and with MNA-SF ( $\mathrm{p}-0.26, \mathrm{p}=0.002)$.

At the univariable binary logistic regression model age (OR $1.07,95 \%$ C.I. $1.04-1.09, \mathrm{p}<0.001)$, the number of chronic therapies at hospital admission (OR 1.24, 95\% C.I. 1.13 - 1.35, $\mathrm{p}<0.001$ ), length of hospital stay (OR 1.07, 95\% C.I. $1.04-1.1$, $\mathrm{p}<0.001)$, ICU stay $(2.61,95 \%$ C.I. $1.03-6.63, \mathrm{p}=0.04)$, NIV use during hospital stay (OR 2.09, 95\% C.I. $1.18-3.70, \mathrm{p}=0.01$ ), days of CRP above the upper normal limit (OR 1.08, 95\% C.I. $1.04-1.11, \mathrm{p}<0.001$ ) and muscle stiffness (OR 1.02, 95\% C.I. $1.01-1.04, \mathrm{p}=0.003)$ resulted significant predictors of probable

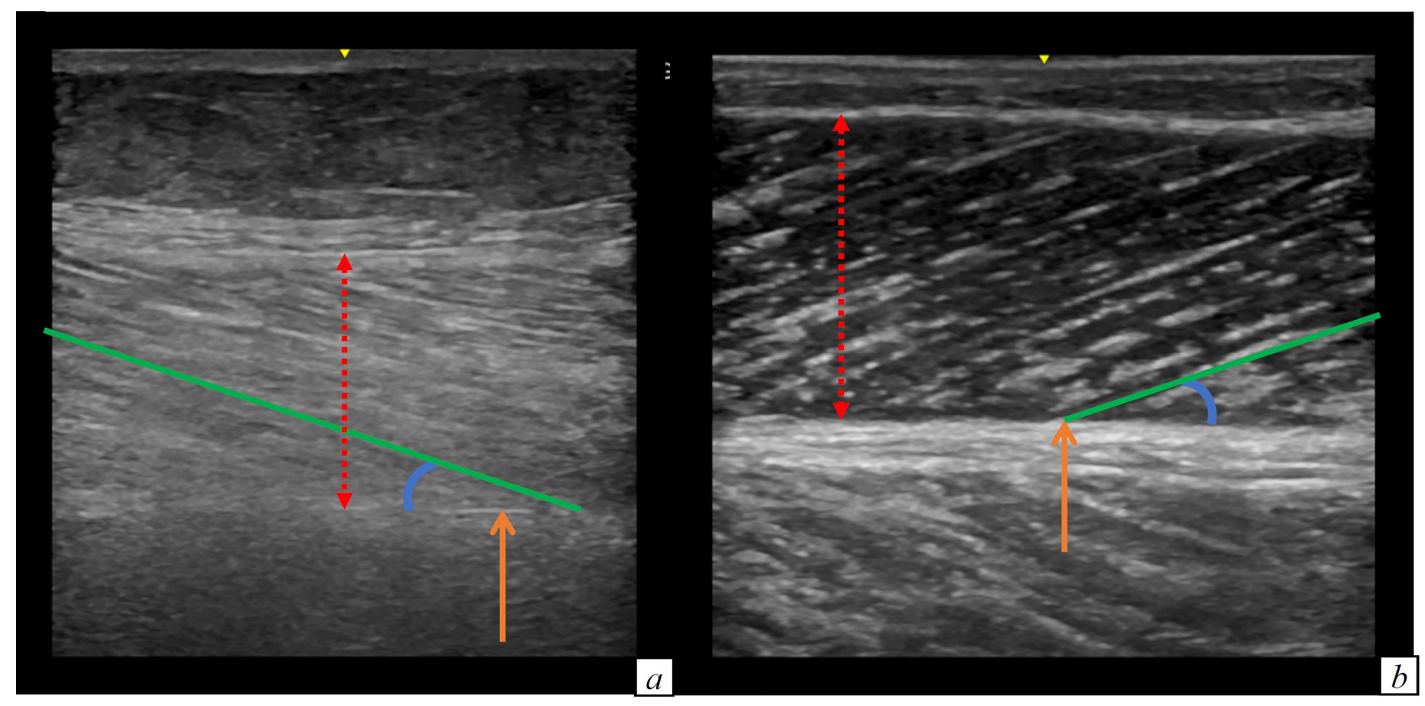

FIGURE 2 | Comparison of the limb ultrasound images of a patient with (A) and without probable sarcopenia (B). (A) Muscle thickness: 1.54 cm, muscle stiffness 127 (B) Muscle thickness: $1.81 \mathrm{~cm}$, muscle stiffness 46.8. RED DOTTED ARROW: muscle thickness; ORANGE ARROW: muscle deep aponeurosis; BLU CIRCLE LINE: pennation angle; GREEN line: muscle fascicle length. 
TABLE 4 | Spearman correlations among muscle ultrasound characteristics, measures of muscle function and nutritional status, age, inflammatory indexes and length of hospital stay.

\begin{tabular}{|c|c|c|c|c|c|c|c|c|c|c|c|c|c|c|}
\hline & & $\begin{array}{c}\text { Muscle } \\
\text { thickness }\end{array}$ & $\begin{array}{c}\text { Pennation } \\
\text { angle }\end{array}$ & $\begin{array}{l}\text { Muscle } \\
\text { stiffness }\end{array}$ & $\begin{array}{l}\text { MNA- } \\
\text { SF }\end{array}$ & SPPB & $\begin{array}{c}\text { Grip } \\
\text { Strength }\end{array}$ & SARCF & $\begin{array}{l}\text { Hospitalization } \\
\text { length }\end{array}$ & $\begin{array}{l}\text { Highest } \\
\text { CRP }\end{array}$ & $\begin{array}{l}\text { Days of } \\
\text { elevated } \\
\text { CRP }\end{array}$ & $\begin{array}{l}\text { Highest } \\
\text { ferritin }\end{array}$ & $\begin{array}{l}\text { Highest } \\
\text { WBC }\end{array}$ & Age \\
\hline \multirow{2}{*}{$\begin{array}{l}\text { Muscle } \\
\text { thickness }\end{array}$} & $p$ & & 0.46 & -0.05 & 0.16 & 0.2 & 0.32 & -0.23 & -0.02 & 0.05 & -0.05 & 0.16 & 0.02 & -0.35 \\
\hline & $p$ & & $<0.001$ & 0.54 & 0.01 & 0.001 & $<0.001$ & $<0.001$ & 0.73 & 0.4 & 0.44 & 0.01 & 0.71 & $\begin{array}{c}< \\
0.001\end{array}$ \\
\hline \multirow{4}{*}{$\begin{array}{l}\text { Pennation } \\
\text { angle } \\
\text { Muscle } \\
\text { stiffness }\end{array}$} & $p$ & 0.46 & & -0.11 & 0.17 & 0.13 & 0.16 & -0.19 & -0.11 & -0.01 & -0.09 & 0.06 & -0.004 & -0.21 \\
\hline & $p$ & $<0.001$ & & 0.17 & 0.008 & 0.03 & 0.01 & 0.005 & 0.07 & 0.9 & 0.15 & 0.33 & 0.95 & 0.001 \\
\hline & $p$ & -0.05 & -0.11 & & -0.26 & -0.11 & -0.26 & 0.3 & 0.15 & 0.04 & 0.13 & -0.13 & 0.003 & 0.22 \\
\hline & $p$ & 0.54 & 0.17 & & 0.002 & 0.19 & 0.001 & $<0.001$ & 0.07 & 0.66 & 0.10 & 0.12 & 0.97 & 0.007 \\
\hline
\end{tabular}

MNA-SF, Mini Nutritional Assessment-Short Form; SPPB, Short Physical Performance Battery; SARC-F, Screening tool for sarcopenia; WBC, White Blood Cells. Bold = statistically significant $(p<0.05)$.

sarcopenia. Muscle stiffness (adjusted OR 1.02, 95\% C.I. 1.002 $1.04, \mathrm{p}=0.03$ ) confirmed to be a significant predictor of probable sarcopenia, in the stepwise multivariable model adjusted for age, sex, NIV, and number of chronic therapies.

Cut-offs for probable sarcopenia identified by the ROC analyses were as follows: $1.51 \mathrm{~cm}$ for muscle thickness (AUC $0.59,95 \%$ C.I. $0.52-0.66 \mathrm{p}=0.017$, sensitivity $41 \%$, specificity $76 \%$ ) and of 73.95 for muscle stiffness (AUC 0.64, 95\% C.I. 0.55 $0.73, \mathrm{p}=0.004$, sensitivity $77 \%$, specificity $48 \%$ ). The results for pennation angle were not statistically significant (AUC 0.55, 95\% C.I. $0.48-0.62, p=0.15)$. ROC curves are illustrated in Figures 1S-3S.

\section{DISCUSSION}

In this observational study, we found that one month after hospital discharge, COVID-19 survivors with reduced muscle function displayed low muscle mass and increased muscle stiffness at the ultrasound evaluation of the dominant medial gastrocnemius as compared with those with normal muscle function. The finding of increased muscle stiffness was confirmed in patients with a pathological SARC-F score too. Moreover, we detected a significant correlation between muscle ultrasound parameters and age, nutritional status and muscle performance. Finally, we detected an association between muscle stiffness and probable sarcopenia and with the ROC analyses we identified the cut-offs of the muscle ultrasound parameters, associated with probable sarcopenia.

Our findings refer to preliminary data, collected one month after hospital discharge. The 3 and 6 months follow ups of the patients of this study are ongoing. Indeed, it is of paramount importance to continue follow up visits over time, because it has been reported that musculoskeletal symptoms can persist 3 and 6 months after hospitalization in COVID-19 survivors (45). Assessing whether these symptoms are underpinned by alteration of muscle function, mass and quality will allow the characterization of the COVID-19 disease on muscles, and the long-term effects of acute sarcopenia, that are presently unknown (46).

Our results on the negative correlation between both muscle thickness and age, and pennation angle and age are in line with the typical architectural remodelling of ageing (47) characterized by decreased muscle size, and reduced pennation angles (48). Indeed, muscle thickness and pennation angle are characteristics of the muscle architecture, that are strongly related one to the other, as previously demonstrated by Kubo (49), and as confirmed in our study.

Detecting changes in muscle architecture is of extreme importance, since these alterations have an impact on the mechanical behaviour of muscles (50). Our study confirmed the presence of a significant correlation between muscle ultrasound characteristics and measures of muscle function. Moreover, we found a significant correlation between muscle ultrasound aspects and nutritional status evaluated with the MNA-SF. Malnutrition, particularly when disease-associated, is known to be associated with alterations in body composition and reductions in fat free mass (51). Both the European Society of Clinical Nutrition and Metabolism (52) and the Global Leadership Initiative on Malnutrition (53) guidelines recommend the evaluation of fat free mass as a diagnostic criterion for malnutrition. Therefore, the association of a malnutrition screening tool with impaired measures of muscle mass and quality is not surprising. Muscle ultrasound is a simple and non-expensive tool to assess skeletal muscle characteristics, and could be a valuable instrument for the screening of malnutrition. Our finding is in line with the study by MateosAngulo et al. (54) that detected an association between MNA-SF and muscle thickness, measured with ultrasonography, in istitutionalized older adults.

Compared to the study of Minetto et al. (55), the median values of muscle thickness were higher in our population (1.7, IQR $1.44-1.93 \mathrm{~cm}$ in the total sample of our study versus $1.42 \pm 0.03 \mathrm{~cm}$ in men and $1.23 \pm 0.28 \mathrm{~cm}$ in women in the study of Minetto). However, Minetto et al. considered a small sample (44 people) of institutionalized pre-frail and frail older adults (mean age $79.2 \pm 8.3$ years) whereas our study included 259 community dwelling people with a median age of 67 years (IQR 56 - 75). Our data on muscle thickness are more in line with the findings of Kubo et al. (49) who detected a mean muscle thickness of $1.93 \pm 0.27 \mathrm{~cm}$ in community dwelling men (mean age $69.5 \pm 4.2$ years) and of $1.77 \pm 0.23 \mathrm{~cm}$ in community dwelling women (mean age $68.0 \pm 5.3$ years).

Differently from Kubo, the median values of the pennation angle, were higher in our sample $\left(22.4^{\circ}\right.$ versus $16.5^{\circ}$ in men and 
$15.6^{\circ}$ in women). Anyway, the measurement of the pennation angle is strongly influenced by the pressure that the sonographer exerts on the muscle, and further data are needed to define normal and pathological values of this parameter in older people.

Our study showed that muscle stiffness is augmented in post COVID-19 patients with reduced muscle function and pathological SARC-F score, as compared with those who had normal values of muscle function and SARC-F. Since we measured muscle stiffness with muscles in a resting condition, our finding refers to passive muscle stiffness. Passive muscle stiffness is an important characteristic, since it regulates the interactions between body and environment. When muscle stiffness is too elevated, the energy of the body-environment interactions can be transmitted to the tissues, causing an injury (56). For example, in people with an elevated muscle stiffness, there is a higher risk of muscle damage after eccentric exercise $(57,58)$.

Passive muscle stiffness is influenced by collagen deposition, inflammation and swelling (59-61). Previous studies showed that the amount of collagen, of advanced glycation end-products and of collagen cross-linking in connective tissue, increase with ageing $(62,63)$. Indeed, we found a significant correlation between muscle stiffness and age. In addition to increasing muscle stiffness [as demonstrated in aged (64) and sarcopenic muscles (65)], the alterations of the extracellular matrix may also favour muscle mass decrease. The alterations in muscle extracellular matrix can alter the regenerative potential of the myogenic progenitor cells (66). However, the exact relation between muscle stiffness and aging has not been clearly elucidated so far. While some studies demonstrated higher muscle stiffness in older people (67-70), others detected opposite results (71). Our findings are in line with the first ones.

In this study we identified possible muscle ultrasound parameters cut-offs, for probable sarcopenia. Muscle ultrasound is a non-invasive, little expensive and low time-consuming technique. As such, it could potentially be considered an optimal screening test for probable sarcopenia. In our study, the muscle thickness cut off for probable sarcopenia had a high specificity (76\%), but a low sensitivity (41\%). It is known that highly specific screening tests unlikely yield false positive results (72). Therefore, people with a pathologic muscle thickness would likely have probable sarcopenia. On the contrary, the ultrasound cut-off for muscle stiffness had a high sensitivity (77\%) but a low specificity (48\%). Highly sensitive screening tests unlikely generate false negative outcomes (72). Thus, people with a normal muscle stiffness would not have probable sarcopenia.

Unfortunately, the AUC of the ROC curves were $<0.7$. These results indicate that muscle ultrasound has a low accuracy in detecting probable sarcopenia, compared to the gold standard hand grip test. Anyway, these results refer to a preliminary and reduced sample, and could be improved by future wider studies. Moreover, muscle ultrasound could be used as a complementary technique to hand grip test to assess the morphologic characteristics of skeletal muscle in patients with probable sarcopenia.

Our study has the merit of having described for the first-time muscle mass and characteristics of post COVID-19 patients with the use of limb muscle ultrasound. Description of muscle ultrasound parameters of post COVID-19 patients with impaired muscle function and pathological SARC-F score is important, since no accepted definition of muscle quality exists so far. Characterizing the changes of muscle architecture through a non-invasive and easy to use tool as echography would provide information to better define muscle quality. Finally, we identified possible cut-off values of the muscle ultrasound parameters suggestive of the risk of sarcopenia in post COVID-19 patients. It could be speculated that muscle ultrasonography may detect subjects slowly recovering from COVID-19, and with potentially negative long-term sequelae.

Some limitations of this study deserve to be mentioned: the relatively limited sample size, the fact that some patients with dementia (in the absence of their care-givers) could have improperly answered to some questions of the SARC-F, the missing information on muscle stiffness for 107 patients, and the dependency on the ability of the operator for the evaluation of muscle mass and quality. Due to the lack of measures of muscle mass/function prior to SARS-CoV-2 infection or during hospitalization, we could not specifically address the impact of COVID-19 on skeletal muscle. However, the main aim of our study was to characterize muscle mass and quality by muscle ultrasound in a population prone to skeletal muscle impairment $(19,20)$, and to assess the association of ultrasound parameters with established tools for the assessment of the risk of sarcopenia. Further studies are needed to assess whether our findings can be generalized to patient populations other than COVID-19 survivors. Finally, an important limit is that we did not compare ultrasound muscle characteristics against reference methods for measuring fat free mass, such as dual-energy Xray absorptiometry, CT or MRI. In the future, wider, multicenter studies will help better define the role of ultrasound for the evaluation of muscle quantity and quality, and correlate these data to relevant clinical outcomes.

In conclusion, we showed that muscle ultrasound parameters have a significant correlation with age, nutritional status and muscle performance in COVID-19 survivors. Although our findings need to be confirmed by studies comparing muscle ultrasound against validated techniques for measuring muscle mass and quality, our study suggests, for the first time, that muscle ultrasound could be an innovative tool to assess muscle mass and quality in COVID-19 survivors.

\section{DATA AVAILABILITY STATEMENT}

The raw data supporting the conclusions of this article will be made available by the authors, without undue reservation.

\section{ETHICS STATEMENT}

The studies involving human participants were reviewed and approved by San Raffaele University Hospital Ethics Committee 
(protocol no. 34/int/2020). The patients/participants provided their written informed consent to participate in this study.

\section{AUTHOR CONTRIBUTIONS}

All authors made substantial contributions to all of the following: (1) the conception and design of the study, or acquisition of data, or analysis and interpretation of data, (2) drafting the article or revising it critically for important intellectual content, (3) final approval of the version to be submitted.

\section{REFERENCES}

1. Cruz-Jentoft AJ, Bahat G, Bauer J, Boirie Y, Bruyère O, Cederholm T, et al. Sarcopenia: Revised European Consensus on Definition and Diagnosis. Age Ageing (2019) 48:16-31. doi: 10.1093/ageing/afy169

2. Cruz-Jentoft AJ, Sayer AA. Sarcopenia. Lancet (2019) 393:2636-46. doi: 10.1016/S0140-6736(19)31138-9

3. De Nardi P, Salandini M, Chiari D, Pecorelli N, Cristel G, Damascelli A, et al. Changes in Body Composition During Neoadjuvant Therapy Can Affect Prognosis in Rectal Cancer Patients: An Exploratory Study. Curr Probl Cancer (2019) 1:100510. doi: 10.1016/j.currproblcancer.2019.100510

4. Pecorelli N, Capretti G, Sandini M, Damascelli A, Cristel G, De Cobelli F, et al. Impact of Sarcopenic Obesity on Failure to Rescue From Major Complications Following Pancreaticoduodenectomy for Cancer: Results From a Multicenter Study. Ann Surg Oncol (2018) 25(1):308-17. doi: 10.1245/s10434-017-6216-5

5. Pecorelli N, Carrara G, De Cobelli F, Cristel G, Damascelli A, Balzano G, et al. Effect of Sarcopenia and Visceral Obesity on Mortality and Pancreatic Fistula Following Pancreatic Cancer Surgery. Br J Surg (2016) 103(4):434-42. doi: $10.1002 /$ bjs. 10063

6. Donini LM, Busetto L, Bauer JM. Critical Appraisal of Definitions and Diagnostic Criteria for Sarcopenic Obesity Based on a Systematic Review. Clin Nutr (2020) 39(8):2368-88. doi: 10.1016/j.clnu.2019.11.024

7. Narici MV, Maffulli N. Sarcopenia: Characteristics, Mechanisms and Functional Significance. Br Med Bull (2010) 95:139e159. doi: 10.1093/bmb/ ldq008

8. McGregor RA, Cameron-Smith D, Poppitt SD. It Is Not Just Muscle Mass: A Review of Muscle Quality, Composition and Metabolism During Ageing as Determinants of Muscle Function and Mobility in Later Life. Longev Healthspan (2014) 3:9. doi: 10.1186/2046-2395-3-9

9. Perkisas S, Lamers S, Degerickx R, Van Mieghem E, Vandewoude M, Verhoeven V, et al. The Relation Between Mortality, Intramuscular Adipose Tissue and Sarcopenia in Hospitalized Geriatric Patients. Eur Geriatr Med (2018) 9:801-7. doi: 10.1007/s41999-018-0110-y

10. Hamaguchi Y, Kaido T, Okumura S, Kobayashi A, Shirai H, Yagi S, et al. Impact of Skeletal Muscle Mass Index, Intramuscular Adipose Tissue Content, and Visceral to Subcutaneous Adipose Tissue Area Ratio on Early Mortality of Living Donor Liver Transplantation. Transplantation (2017) 101:565-74. doi: 10.1097/TP.0000000000001587

11. Reinders I, Murphy RA, Brouwer IA, Visser M, Launer L, Siggeirsdottir K, et al. Muscle Quality and Myosteatosis: Novel Associations With Mortality Risk: The Age, Gene/Environment Susceptibility (AGES)-Reykjavik Study. Am J Epidemiol (2016) 183:53-60. doi: 10.1093/aje/kwv153

12. Heymsfield SB, Gonzalez MC, Lu J, Jia G, Zheng J. Skeletal Muscle Mass and Quality: Evolution of Modern Measurement Concepts in the Context of Sarcopenia. Proc Nutr Soc (2015) 74:355-66. doi: 10.1017/ S0029665115000129

13. Lynch NA, Metter EJ, Lindle RS, Fozard JL, Tobin JD, Roy TA, et al. Muscle Quality. IAge-Associated Differences Between Arm and Leg Muscle Groups. J Appl Physiol (1985) (1999) 86:188-94. doi: 10.1152/jappl.1999.86.1.188

14. Rolland Y, Lauwers-Cances V, Pahor M, Fillaux J, Grandjean H, Vellas B, et al. Muscle Strength in Obese Elderly Women: Effect of Recreational Physical

\section{FUNDING}

This study was financially supported by Ministero della Salute, Italy, and by COVID-19 donations.

\section{SUPPLEMENTARY MATERIAL}

The Supplementary Material for this article can be found online at: https:/www.frontiersin.org/articles/10.3389/fendo.2022.801133/ full\#supplementary-material

Activity in a Cross-Sectional Study. Am J Clin Nutr (2004) 79:552-7. doi: 10.1093/ajcn/79.4.552

15. Tracy BL, Ivey FM, Hurlbut D, Martel GF, Lemmer JT, Siegel EL, et al. Muscle Quality. II. Effects Of Strength Training in 65- to 75-Yr-Old Men and Women. J Appl Physiol (1985) (1999) 86:195-201. doi: 10.1152/ jappl.1999.86.1.195

16. Welch C, Greig C, Masud T, Wilson D, Jackson TA. COVID-19 and Acute Sarcopenia. Aging Dis (2020) 11(6):1345-51. doi: 10.14336/AD.2020.1014

17. Pironi L, Sasdelli AS, Ravaioli F, Baracco B, Battaiola C, Bocedi G, et al. Malnutrition and Nutritional Therapy in Patients With SARS-CoV-2 Disease. Clin Nutr (2021) 40(3):1330-7. doi: 10.1016/j.clnu.2020.08.021

18. Di Filippo L, De Lorenzo R, D'Amico M. COVID-19 is Associated With Clinically Significant Weight Loss and Risk of Malnutrition, Independent of Hospitalisation: A Post-Hoc Analysis of a Prospective Cohort Study. Clin Nutr (2021) 40(4):2420-6. doi: 10.1016/j.clnu.2020.10.043

19. Paneroni M, Simonelli C, Saleri M, Bertacchini L, Venturelli M, Troosters T, et al. Muscle Strength and Physical Performance in Patients Without Previous Disabilities Recovering From COVID-19 Pneumonia. Am J Phys Med Rehabil (2021) 100:105-9. doi: 10.1097/PHM.0000000000001641

20. Wierdsma NJ, Kruizenga HM, Konings LA, Krebbers D, Jorissen J, Joosten MHI, et al. Poor Nutritional Status, Risk of Sarcopenia and Nutrition Related Complaints Are Prevalent in COVID-19 Patients During and After Hospital Admission. Clin Nutr ESPEN (2021) 43:369-76. doi: 10.1016/j.clnesp. 2021.03.021

21. Piotrowicz K, Gasowski J, Michel JP, Veronese N. Post-COVID-19 Acute Sarcopenia: Physiopathology and Management. Aging Clin Exp Res (2021) 33 (10):2887-98.

22. Disser NP, De Micheli AJ, Schonk MM, Konnaris MA, Piacentini AN, Edon DL, et al. Musculoskeletal Consequences of COVID-19. J Bone Joint Surg Br (2020) 102:1197-204. doi: 10.2106/JBJS.20.00847

23. Bagnato S, Boccagni C, Marino G, Prestandrea C, D’Agostino T, Rubino F. Critical Illness Myopathy After COVID-19. Int J Infect Dis (2020) 99:276-78. doi: 10.1016/j.ijid.2020.07.072

24. Wilson D, Jackson T, Sapey E, Lord JM. Frailty and Sarcopenia: The Potential Role of an Aged Immune System. Ageing Res Rev (2017) 36:1-10. doi: 10.1016/ j.arr.2017.01.006

25. Clegg A, Young J, Iliffe S, Rikkert MO, Rockwood K. Frailty in Elderly People. Lancet (2013) 381:752-62. doi: 10.1016/S0140-6736(12)62167-9

26. Rocheteau P, Chatre L, Briand D, Mebarki M, Jouvion G, Bardon J, et al. Sepsis Induces Long-Term Metabolic and Mitochondrial Muscle Stem Cell Dysfunction Amenable by Mesenchymal Stem Cell Therapy. Nat Commun (2015) 6:10145. doi: 10.1038/ncomms10145

27. Heymsfield SB, Adamek M, Gonzalez MC, Jia G, Thomas DM, et al. Assessing Skeletal Muscle Mass: Historical Overview and State of the Art. J Cachexia Sarcopenia Muscle (2014) 5:9e18. doi: 10.1007/s13539-014-0130-5

28. Narici MV, Binzoni T, Hiltbrand E, Fasel J, Terrier F, Cerretelli P. In Vivo Human Gastrocnemius Architecture With Changing Joint Angle at Rest and During Graded Isometric Contraction. J Physiol (Lond) (1996) 496:287-97. doi: 10.1113/jphysiol.1996.sp021685

29. Cartwright MS, Demar S, Griffin LP, Balakrishnan N, Harris JM, Walker FO, et al. Validity and Reliability of Nerve and Muscle Ultrasound. Muscle Nerve (2013) 47(4):515-21. doi: 10.1002/mus.23621 
30. Scott JM, Martin DS, Ploutz-Snyder R, Caine T, Matz T, Arzeno NM, et al. Reliability and Validity of Panoramic Ultrasound for Muscle Quantification. Ultrasound Med Biol (2012) 38(9):1656-61. doi: 10.1016/j.ultrasmedbio.2012. 04.018

31. Muscaritoli M, Anker SD, Argilés J, Aversa Z, Bauer JM, Biolo G, et al. Consensus Definition of Sarcopenia, Cachexia and Pre-Cachexia: Joint Document Elaborated by Special Interest Groups (SIG) "Cachexia-Anorexia in Chronic Wasting Diseases" and "Nutrition in Geriatrics". Clin Nutr (2010) 29:154e159. doi: 10.1016/j.clnu.2009.12.004

32. Fielding RA, Vellas B, Evans WJ, Bhasin S, Morley JE, Newman AB, et al. Sarcopenia: An Undiagnosed Condition in Older Adults. Current Consensus Definition: Prevalence, Etiology, and Consequences. J Am Med Dir Assoc (2011) 12:249e256. doi: 10.1016/j.jamda.2011.01.003

33. Ticinesi A, Meschi T, Narici MV, Lauretani F, Maggio M. Muscle Ultrasound and Sarcopenia in Older Individuals: A Clinical Perspective. J Am Med Dir Assoc (2017) 18(4):290-300. doi: 10.1016/j.jamda.2016.11.013

34. Rovere Querini P, De Lorenzo R, Conte C. Post-COVID-19 Follow-Up Clinic: Depicting Chronicity of a New Disease. Acta BioMed (2020) 91(9-S):22-8. doi: 10.23750/abm.v91i9-S.10146

35. Rovere-Querini P, Tresoldi C, Conte C, Ruggeri A, Ghezzi S, De Lorenzo R, et al. Biobanking for COVID-19 Research. Panminerva Med (2020). doi: $10.23736 / \mathrm{S} 0031-0808.20 .04168-3$

36. Malmstrom TK, Morley JE. SARC-F: A Simple Questionnaire to Rapidly Diagnose Sarcopenia. JAMDA (2013) 14:531.e532. doi: 10.1016/j.jamda. 2013.05.018

37. Dodds RM, Syddall HE, Cooper R, Benzeval M, Deary IJ, Dennison EM, et al. Grip Strength Across the Life Course: Normative Data From Twelve British Studies. PloS One (2014) 9:e113637. doi: 10.1371/journal.pone.0113637

38. Guralnik JM, Ferrucci L, Simonsick EM, Salive ME, Wallace RB. LowerExtremity Function in Persons Over the Age of 70 Years as a Predictor of Subsequent Disability. N Engl J Med (1995) 332:556-61. doi: 10.1056/ NEJM199503023320902

39. Rubenstein LZ, Harker JO, Salva A, Guigoz Y, Vellas B. Screening for Undernutrition in Geriatric Practice: Developing the Short-Form MiniNutritional Assessment (MNA-SF) 1. J Gerontol (2001) 56:M366-72. doi: 10.1093/gerona/56.6.M366

40. Sipila S, Suominen H. Muscle Ultrasonography and Computed Tomography in Elderly Trained and Untrained Women. Muscle Nerve (1993) 16:294-300. doi: 10.1002/mus.880160309

41. Ismail C, Zabal J, Hernandez HJ, Woletz P, Manning H, Teixeira C, et al. Diagnostic Ultrasound Stimates of Muscle Mass and Muscle Quality Discriminate Between Women With and Without Sarcopenia. Front Physiol (2015) 6:302. doi: 10.3389/fphys.2015.00302

42. Phan A, Lee J, Gao J. Ultrasound Shear Wave Elastography in Assessment of Skeletal Muscle Stiffness in Senior Volunteers. Clin Imaging (2019) 58:22-6. doi: 10.1016/j.clinimag.2019.06.006

43. Arts IM, Pillen S, Schelhaas HJ, Overeem S, Zwarts MJ. Normal Values 441 for Quantitative Muscle Ultrasonography in Adults. Muscle Nerve (2010) 41:3241. doi: $10.1002 /$ mus. 21458

44. Caresio C, Molinari F, Emanuel G, Minetto MA. Muscle Echo Intensity: Reliability and Conditioning Factors. Clin Physiol Funct Imaging (2015) 35:393-403. doi: 10.1111/cpf.12175

45. Karaarslan F, Demircioğlu Güneri F, Kardeş S. Long COVID: Rheumatologic/ Musculoskeletal Symptoms in Hospitalized COVID-19 Survivors at 3 and 6 Months. Clin Rheumatol (2022) 41(1):289-96. doi: 10.1007/s10067-02105942-x

46. Welch C, Hassan-Smith ZK, Greig CA, Lord JM, Jackson TA. Acute Sarcopenia Secondary to Hospitalisation - An Emerging Condition Affecting Older Adults. Aging Dis (2018) 9:151-64. doi: 10.14336/AD.2017.0315

47. Morse C, Thom J, Birch K, Narici M. Changes in Triceps Surae Muscle Architecture With Sarcopenia. Acta Physiol Scand (2005) 183(3):291-8. doi: 10.1111/j.1365-201X.2004.01404.x

48. Narici M, Maganaris C, Reeves N, Capodaglio P. Effect of Aging on Human Muscle Architecture. J Appl Physiol (2003) 95(6):2229-34. doi: 10.1152/ japplphysiol.00433.2003

49. Kubo K, Kanehisa H, Azuma K, Ishizu M, Kuno S-Y, Okada M, et al. Muscle Architectural Characteristics in Young and Elderly Men and Women. Int $J$ Sports Med (2003) 24:125-30. doi: 10.1055/s-2003-38204
50. Lieber RL, Friden J. Functional and Clinical Significance of Skeletal Muscle Architecture. Muscle Nerve (2000) 23:1647-66. doi: 10.1002/1097-4598 (200011)23:11<1647::AID-MUS1>3.0.CO;2-M

51. Sobotka L ed. Basics in Clinical Nutrition. 4th ed. Prague: Galen (2012).

52. Cederholm T, Bosaeus I, Barazzoni R, Bauer J, Van Gossum A, Klek S, et al. Diagnostic Criteria for Malnutrition-An ESPEN Consensus Statement. Clin Nutr (2015) 34:335-40. doi: 10.1016/j.clnu.2015.03.001

53. Cederholm T, Jensen GL, Correia MITD, Gonzalez M, Fukushima R, Higashiguchi T, et al. GLIM Criteria for the Diagnosis of Malnutrition - A Consensus Report From the Global Clinical Nutrition Community. J Cachexia Sarcopenia Muscle (2019) 10:207-17. doi: 10.1002/jcsm.12383

54. Mateos-Angulo A, Galán-Mercant A, Cuesta-Vargas AI. Ultrasound Muscle Assessment and Nutritional Status in Institutionalized Older Adults: A Pilot Study. Nutrients (2019) 11(6):1247. doi: 10.3390/nu11061247

55. Minetto MA, Caresio C, Menapace T, Hajdarevic A, Marchini A, Molinari F, et al. Ultrasound-Based Detection of Low Muscle Mass for Diagnosis of Sarcopenia in Older Adults. PM R (2016) 8(5):453-62. doi: 10.1016/ j.pmrj.2015.09.014

56. Blanpied P, Smidt GL. The Difference in Stiffness of the Active Plantarflexors Between Young and Elderly Human Females. J Gerontol (1993) 48(2):M5863. doi: 10.1093/geronj/48.2.M58

57. McHugh MP, Connolly DA, Eston RG, Kremenic IJ, Nicholas SJ, Gleim GW, et al. The Role of Passive Muscle Stiffness in Symptoms of Exercise-Induced Muscle Damage. Am J Sports Med (1999) 27:594-9. doi: 10.1177/ 03635465990270050801

58. Miyamoto N, Hirata K, Miyamoto-Mikami E, Yasuda O, Kanehisa H. Associations of Passive Muscle Stiffness, Muscle Stretch Tolerance, and Muscle Slack Angle With Range of Motion: Individual and Sex Differences. Sci Rep (2018) 8:8274. doi: 10.1038/s41598-018-26574-3

59. Brandenburg JE, Eby SF, Song P, Zhao H, Brault JS, Chen S, et al. Ultrasound Elastography: The New Frontier in Direct Measurement of Muscle Stiffness. Arch Phys Med Rehabil (2014) 95:2207-19. doi: 10.1016/j.apmr.2014.07.007

60. Hug F, Tucker K, Gennisson JL, Tanter M, Nordez A. Elastography for Muscle Biomechanics: Toward the Estimation of Individual Muscle Force. Exerc Sport Sci Rev (2015) 43:125-33. doi: 10.1249/JES.00000000000000049

61. Creze M, Nordez A, Soubeyrand M, Rocher L, Maitre X, Bellin MF. Shear Wave Sonoelastography of Skeletal Muscle: Basic Principles, Biomechanical Concepts, Clinical Applications, and Future Perspectives. Skeletal Radiol (2018) 47:457-71. doi: 10.1007/s00256-017-2843-y

62. Alnaqueeb MA, AI Zaid NS, Goldspink G. Connective Tissue Changes and Physical Properties of Developing and Ageing Skeletal Muscle. J Anat (1984) 139:677-89.

63. Kovanen V, Suominen H, Heikkinen E. Mechanical Properties of Fast and Slow Skeletal Muscle With Special Reference to Collagen and Endurance Training. J Biomech (1984) 17:725-35. doi: 10.1016/0021-9290(84)90103-9

64. Wood LK, Kayupov E, Gumucio JP, Mendias CL, Claflin DR, Brooks SV. Intrinsic Stiffness of Extracellular Matrix Increases With Age in Skeletal Muscles of Mice. Journal of Applied Physiology. J Appl Physiol (1985) 117 (4):363-9. doi: 10.1152/japplphysiol.00256.2014

65. Brashear SE, Wohlgemuth RP, Gonzalez G. Passive Stiffness of Fibrotic Skeletal Muscle in Mdx Mice Relates to Collagen Architecture. J Physiol (2021) 599(3):943-62. doi: 10.1113/JP280656

66. Lacraz G, Rouleau AJ, Couture V, Söllrald T, Drouin G, Veillette N, et al. Increased Stiffness in Aged Skeletal Muscle Impairs Muscle Progenitor Cell Proliferative Activity. PloS One (2015) 10(8):e0136217. doi: 10.1371/ journal.pone. 0136217

67. Eby SF, Cloud BA, Brandenburg JE, Giambini H, Song P, Chen S, et al. Shear Wave Elastography of Passive Skeletal Muscle Stiffness: Influences of Sex and Age Throughout Adulthood. Clin Biomech (Bristol Avon) (2015) 30:22-7. doi: 10.1016/j.clinbiomech.2014.11.011

68. Saito A, Wakasa M, Kimoto M, Ishikawa T, Tsugaruya M, Kume Y, et al. AgeRelated Changes in Muscle Elasticity and Thickness of the Lower Extremities Are Associated With Physical Functions Among Communitydwelling Older Women. Geriatr Gerontol Int (2019) 19:61-5. doi: 10.1111/ggi.13567

69. Ochala J, Frontera WR, Dorer DJ, Van Hoecke J, Krivickas L. Single Skeletal Muscle Fiber Elastic and Contractile Characteristics in Young and Older Men. J Gerontol A Biol Sci Med Sci (2007) 62(4):375-81. doi: 10.1093/gerona/ 62.4 .375 
70. Gao Y, Kostrominova TY, Faulkner JA, Wineman AS. Age-Related Changes in the Mechanical Properties of the Epimysium in Skeletal Muscles of Rats. J Biomechan (2008) 41:465-9. doi: 10.1016/ j.jbiomech.2007.09.021

71. Akagi R, Yamashita Y, Ueyasu Y. Age-Related Differences in Muscle Shear Moduli in the Lower Extremity. Ultrasound Med Biol (2015) 41:2906-12. doi: 10.1016/j.ultrasmedbio.2015.07.011

72. Trevethan R. Sensitivity, Specificity, and Predictive Values: Foundations, Pliabilities, and Pitfalls in Research and Practice. Front Public Health (2017) 5:307. doi: 10.3389/fpubh.2017.00307

Conflict of Interest: The authors declare that the research was conducted in the absence of any commercial or financial relationships that could be construed as a potential conflict of interest.
Publisher's Note: All claims expressed in this article are solely those of the authors and do not necessarily represent those of their affiliated organizations, or those of the publisher, the editors and the reviewers. Any product that may be evaluated in this article, or claim that may be made by its manufacturer, is not guaranteed or endorsed by the publisher.

Copyright (C) 2022 Damanti, Cilla, Tuscano, De Lorenzo, Manganaro, Merolla Pacioni, Pomaranzi, Tiraferri, Martinenghi, Vitali, Bosi, Conte, Giustina, Tresoldi and Rovere Querini. This is an open-access article distributed under the terms of the Creative Commons Attribution License (CC BY). The use, distribution or reproduction in other forums is permitted, provided the original author(s) and the copyright owner(s) are credited and that the original publication in this journal is cited, in accordance with accepted academic practice. No use, distribution or reproduction is permitted which does not comply with these terms. 